\title{
Tumor-suppressing effects of microRNA-612 in bladder cancer cells by targeting malic enzyme 1 expression
}

\author{
MENGNAN LIU $^{1,2^{*}}$, YIFAN CHEN ${ }^{1 *}$, BISHENG HUANG ${ }^{1.2}$, SHIYU MAO $^{1}$, \\ $\mathrm{KEKE} \mathrm{CAI}^{1}$, LONGSHENG WANG ${ }^{1}$ and XUDONG YAO ${ }^{1}$ \\ ${ }^{1}$ Anhui Medical University, Hefei, Anhui 230601; ${ }^{2}$ Department of Urinary Surgery, \\ Shanghai Tenth People's Hospital, School of Medicine, Tongji University, Shanghai 200072, P.R. China
}

Received November 10, 2017; Accepted March 28, 2018

DOI: 10.3892/ijo.2018.4342

\begin{abstract}
The present study investigated the possible tumor-suppressing function of microRNA (miR)-612 and the underlying molecular mechanism of its action in bladder cancer in vitro and in vivo. Reverse transcription-quantitative polymerase chain reaction (RT-qPCR) was carried out to quantify the expression levels of miR-612 in bladder cancer tissues and cell lines. The data demonstrated that the level of miR-612 expression was significantly reduced in bladder cancer tissues and cell lines, as compared with that in non-cancerous tissues and cells. Reduced miR-612 expression was associated with advanced tumor, lymph node and metastasis stages, and with distant metastasis of bladder cancer. A functional study revealed that transfection of cells with an miR-612 mimic suppressed bladder cancer cell growth, colony formation, migration, invasion and epithelialmesenchymal transition. Bioinformatics analysis identified that miR-612 targeted the expression of malic enzyme 1 (ME1), and this was confirmed by western blot and luciferase reporter assay results. Furthermore, the ME1 expression levels were inversely associated with miR-612 expression in bladder cancer tissue specimens. In addition, knockdown of ME1 expression using ME1 siRNA mimicked the effect of ectopic miR-612 overexpression in bladder cancer cells in terms of tumor cell growth, migration and invasion. By contrast, ME1 overexpression weakened the inhibitory effect of the miR-612 mimic in bladder cancer cells. In conclusion, the present study demonstrated that miR-612 may function as a tumor suppressor in bladder cancer by targeting ME1 expression.
\end{abstract}

Correspondence to: Dr Xudong Yao or Dr Mengnan Liu, Department of Urinary Surgery, Shanghai Tenth People's Hospital, School of Medicine, Tongji University, 301 Yanchang Road, Zhabei, Shanghai 200072, P.R. China

E-mail: yaoxudong67@sina.com

E-mail: liumengnan2015@163.com

*Contributed equally

Key words: microRNA-612, bladder cancer, malic enzyme 1

\section{Introduction}

Bladder cancer is a common malignancy of the urinary system, and is clinically characterized by a high recurrence rate and rapid progression (1). An estimated 429,800 new bladder cancer cases and 165,100 cancer-associated mortalities occurred in 2012 worldwide (2). In United States, an estimated 79,030 new bladder cancer cases and 16,870 bladder cancer-associated mortalities were reported in 2017 (3). Histologically, 75\% of cases with non-muscle-invasive bladder cancer result in a favorable prognosis; however, the remaining $25 \%$ of cases with muscle-invasive bladder cancer exhibit a poor prognosis (4-6). To date, the molecular mechanism underlying bladder cancer oncogenesis remains not fully defined; therefore, the molecular mechanisms underlying the carcinogenic function and progression of bladder cancer should be urgently investigated. This research will help to develop novel approaches for early detection, prediction of prognosis and effective therapy for invasive bladder cancer, as well as provide a novel method for bladder cancer prevention, eventually improving the prognosis and reducing the incidence of bladder cancer.

MicroRNAs (miRNAs or miRs) are a class of non-coding small RNA molecules of approximately 22 nucleotides in length, which possess the capacity of oncogenic or anti-oncogenic activity by binding to the 3'-untranslated regions (UTRs) of their target mRNA molecules. miRNAs negatively regulate the expression of their target genes and serve a vital role in the manipulation of human diseases and tumorigenesis (7). Accumulating studies have indicated that miRNAs function to regulate various physiological and pathological processes, including cell growth, migration, invasion, cell cycle distribution, cell differentiation, apoptosis, oncogenesis, lipid metabolism and drug resistance $(8,9)$. Since alterations in miRNA expression are associated with the development and progression of bladder cancer, certain miRNAs may serve as potential diagnostic markers or therapeutic strategies for bladder cancer (10-14). For instance, Zhang et al (15) have observed that miR-497 expression is able to inhibit bladder cancer cell proliferation, migration and invasion. In addition, Yu et al (16) have demonstrated that miR-613 significantly inhibits bladder cancer cell proliferation, migration and invasion. Wu et al (17) have also reported that miR-429 overexpression can reverse epithelial-mesenchymal transition (EMT) 
by restoring the expression of E-cadherin in bladder cancer. The present study focuses on miR-612, which has recently been reported to be downregulated in numerous types of human cancer, and functions as a putative tumor suppressor in hepatocellular carcinoma $(18,19)$ and colorectal cancer $(20)$.

In the present study, the level of miR-612 expression in bladder cancer tissues was investigated, and its association with the clinicopathological data of patients was determined. The effects of miR-612 expression in bladder cancer cells in vitro and in nude mouse xenografts were then assessed, while bioinformatics analysis was performed to identify and confirm the miR-612 target genes. The present study is expected to provide novel insightful information regarding miR-612 in bladder cancer development and progression to assist in further investigation of this miRNA as a novel tumor biomarker or therapeutic strategy for bladder cancer in the future.

\section{Materials and methods}

Patients and tissue specimens. Bladder cancer and adjacent non-cancerous tissue (ANT) specimens were collected from 46 patients who underwent surgical resection of tumor lesions at the Shanghai Tenth People's Hospital of Tongji University (Shanghai, China) between January 2012 and December 2015. The clinical characteristics of these patients are shown in Table I. None of the patients had received any chemotherapy or radiotherapy prior to surgery. Following surgery, tissue specimens were immediately placed into liquid nitrogen or were snap-frozen in liquid nitrogen, and stored at $-80^{\circ} \mathrm{C}$ until further use. All patients were diagnosed with invasive bladder cancer according to the 2002 version of the American Joint Committee on Cancer/Union for International Cancer Control tumor, lymph node and metastasis (TNM) staging system (21). This study was approved by the Ethics Committee of Shanghai Tenth People's Hospital of Tongji University, and informed consent was obtained from all patients or their relatives.

Target gene prediction of miR-612. To predict the target genes of miR-612 we conducted a bioinformatics analysis using a panel of different algorithms including DIANAmT (http:// www.microrna.gr/microT) miRanda (http://www.microrna. org/microrna/home.do) miRDB (http://www.mirdb.org/) PICTAR5 (http://www.pictar.org/) RNA22 (http://cbcsrv. watson.ibm.com/rna22.html) TargetScan (http://www. targetscan.org/) and miRWalk (http://zmf.umm.uni-heidelberg.de/apps/zmf/mirwalk2/).

miRWalk 2.0 is an updated version of the database miRWalk that can used to validated miRNAs binding sites from the human, mouse and rat on their target genes (http://zmf.umm.uniheidelberg.de/apps/zmf/mirwalk2/) $(22,23)$. MiRWalk2.0 not only recorded the gene sequence of micrornas binding sites, but also combines this information with a comparison of binding sites resulting from 12 existing miRNA-target prediction programs (DIANA-microTv4.0, DIANA-microT-CDS, miRanda-rel2010, mirBridge, miRDB4.0, miRmap, miRNAMap, doRiNA i.e., PicTar2, PITA, RNA22v2, RNAhybrid 2.1 and Targetscan 6.2), we predicted 21 possible target genes in 7 databases (DIANAmT, miRanda, miRDB, miRWalk PICTAR5, RNA22 and Targetscan). Subsequently, based on the combined score of mirna-612 on Targetscan, 9 possible target genes above
95 score were selected (LRRC41, DUSP14, ZNF318, GABRA5, SMAD6, YTHDF1, HOXA13, TSG101 and ME1).

To further narrow the screening range, we retrieved the 9 genes on PubMed by keywords '(Tumor) AND LRRC41', '(Tumor) AND DUSP14', '(Tumor) AND ZNF318', '(Tumor) AND GABRA5', '(Tumor) AND SMAD6', '(Tumor) AND YTHDF1', '(Tumor) AND HOXA13', '(Tumor) AND TSG101', '(Tumor) AND ME1', excluding the genes that have been studied in bladder cancer (HOXA13\{Hu, 2017 \#120\} (24) and no high expression in other tumors [LRRC41 (25), DUSP14 (26), ZNF318 (27), GABRA5 (28)].

Cell lines and culture. Human bladder cancer T24, UMUC3, 5637 and J82 cell lines, and the immortalized human normal bladder epithelial cell line SV-HUC-1 were obtained from type culture collection of the Chinese Academy of Sciences (Shanghai, China). SV-HUC-1 cells were maintained in F12K medium (Sigma-Aldrich; Merck KGaA, Darmstadt, Germany), while T24, UMUC3 and 5637 cells were cultured in RPMI-1640 medium (Gibco; Thermo Fisher Scientific, Inc., Waltham, MA, USA) and J82 cells were cultured in Dulbecco's modified Eagle's medium (Gibco; Thermo Fisher Scientific, Inc.). Cells were cultured at $37^{\circ} \mathrm{C}$ in a humidified incubator with $5 \% \mathrm{CO}_{2}$. All cell culture media were supplemented with $10 \%$ fetal bovine serum (FBS; Gibco; Thermo Fisher Scientific, Inc.) and $1 \%$ penicillin/streptomycin (Hyclone; GE Healthcare Life Sciences, Logan, UT, USA).

Transient gene transfection. Human miR-612 mimic (miR-612) and the corresponding negative control mimic (miR-NC), as well as malic enzyme 1 siRNA (si-ME1) and the corresponding negative control siRNA (si-NC), were purchased from GenePharma Co., Ltd. (Shanghai, China). A plasmid carrying malic enzyme 1 (ME1) cDNA was purchased from RiboBio Co., Ltd. (Guangzhou, China). For transient gene transfection, bladder cancer T24 cells were grown and transiently transfected with these reagents using Lipofectamine $\AA$ 3000 (Invitrogen; Thermo Fisher Scientific, Inc.), according to the manufacturer's protocol for $6 \mathrm{~h}$ at $37^{\circ} \mathrm{C}$. The cells were then subjected to different assays.

RNA isolation and reverse transcriptase-quantitative polymerase chain reaction ( $R T-q P C R)$. Total RNA was isolated from frozen tissues or cultured cells using TRIzol reagent (Invitrogen; Thermo Fisher Scientific, Inc.), according to the manufacturer's protocol. The concentration and purity of the RNA samples were measured by a NanoDrop 2000 spectrophotometer (Thermo Fisher Scientific, Inc.). For detection of miR-612, $1 \mu \mathrm{g}$ total RNA was reverse transcribed into cDNA using a One Step Prime script miRNA cDNA Synthesis kit (Qiagen, Inc., Valencia, CA, USA), and qPCR was performed using the KAPA SYBR FAST qPCR kit (Kapa Biosystems, Inc., Boston, MA, USA), with U6 mRNA used to normalize the miR-612 expression level. The primers used were as follows: miR-612 forward, 5-GCAGGGCTTCTGAGCTCCTTAA-3, U6 forward, 5-CAAATTCGTGAAGCGTTCCATAT-3; the common reverse primer was purchased from RiboBio Co., Ltd. The amplification procedure was as follows: $5 \mathrm{~min}$ at $95^{\circ} \mathrm{C}$, followed by 40 cycles at $95^{\circ} \mathrm{C}$ for $30 \mathrm{sec}$ and $65^{\circ} \mathrm{C}$ for $45 \mathrm{sec}$. 
Table I. Clinicopathological characteristics of the patients with bladder cancer.

\begin{tabular}{lc}
\hline Variables & No. of cases \\
\hline Age (years) & \\
$<60$ & 38 \\
$\geq 60$ & \\
Sex & 41 \\
Male & 5 \\
Female & \\
TNM stage & 19 \\
T1-T2 & 27 \\
T3-T4 & \\
Distant metastasis & 32 \\
No & 14 \\
Yes & \\
\hline
\end{tabular}

TNM, tumor-node-metastasis.

For detection of the ME1 mRNA level, cDNA was synthesized using the PrimeScript RT Reagent kit (Takara Bio, Inc., Tokyo, Japan), according to the manufacturer's protocol. qPCR for mRNA detection was also performed using the KAPA SYBR FAST qPCR kit (Kapa Biosystems, Inc.), The primers used were as follows: ME1 forward, 5'-GCAGGTCT CCTTGCAGCTCT-3' and reverse, 5'-TCCAAGGCCATCACA ATCAG-3'; GAPDH forward, 5'-ATGTCGTGGAGTCTACT GGC-3' and reverse, 5'-TGACCTTGCCCACAGCCTTG-3'. The PCR parameters for relative quantification were as follows: $2 \mathrm{~min}$ at $95^{\circ} \mathrm{C}$, followed by 40 cycles of $45 \mathrm{sec}$ at $57^{\circ} \mathrm{C}$ and $45 \mathrm{sec}$ at $72^{\circ} \mathrm{C}$. The ME1 mRNA level was normalized to that of GAPDH. The relative expression levels of miR-612 and ME1 were analyzed by the $2^{-\Delta \Delta \mathrm{Cq}} \operatorname{method}(29)$.

Cell proliferation and colony formation assays. The cell proliferation rate was measured using the Cell Counting Kit-8 (CCK-8; Dojindo Molecular Technologies, Inc., Kumamoto, Japan). Briefly, the transfected cells were seeded into 96-well plates at a density of $1 \times 10^{3}$ cells/well and grown for up to 5 days. Subsequently, $10 \mu \mathrm{l}$ CCK- 8 reagent was added into each well, and the cells were further incubated at $37^{\circ} \mathrm{C}$ for $2 \mathrm{~h}$. Next, the optical density value was measured at $450 \mathrm{~nm}$ using a microplate spectrophotometer obtained from BioTek Instruments, Inc. (Winooski, VT, USA).

For the tumor cell colony formation assay, 1,000 transfected cells/well were seeded into 6-well plates and cultured for 14 days. Subsequent to culturing, the cell colonies were washed three times with phosphate-buffered saline (PBS), then fixed with $75 \%$ ethanol and stained with $0.1 \%$ crystal violet solution. Cell colonies with $\geq 50$ cells were counted under a light microscope (Olympus Corporation, Tokyo, Japan). The experiments were performed in triplicate and repeated at least once.

Cell migration and invasion assays. Transwell chambers (Corning, Inc., Lowell, MA, USA) containing 6.5-mm-diameter polycarbonate filters with an $8-\mu \mathrm{m}$ pore size were used to measure the cell migration capacity. Briefly, $5 \times 10^{4}$ transfected cells in RPMI-1640 medium (200 $\mu$ l) without FBS were seeded into each insert (24-well plates), and $600 \mu 1$ cell culture medium containing $10 \%$ FBS was added to the bottom chamber. The cells were allowed to migrate for $16 \mathrm{~h}$ at $37^{\circ} \mathrm{C}$ and $5 \% \mathrm{CO}_{2}$. For the tumor cell invasion assay, the filter of the Transwell chamber was precoated with Matrigel (BD Biosciences, Franklin Lakes, NJ, USA), and the cells were allowed to invade for $24 \mathrm{~h}$. The remaining experimental procedures were the same as those of the tumor cell migration assay. At the end of the experiments, the cells remaining in the upper inserts were carefully removed using a cotton swab, whereas cells that had migrated or invaded into the reverse side of the filters were fixed with $70 \%$ ethanol for $30 \mathrm{~min}$ and stained with $0.1 \%$ crystal violet for $10 \mathrm{~min}$. Images of five randomly selected microscopic fields were captured under a microscope (Olympus Corporation), and then the number of cells was counted. The experiments were performed in triplicate and repeated at least once.

Luciferase reporter assay. The wild-type 3'-UTR segment and a mutant 3'-UTR segment of ME1 cDNA were amplified using PCR, and then inserted into the luciferase gene using the psiCHECK-2 vector (Promega Corporation, Madison, WI, USA), according to the manufacturer's instructions. Luciferase assay was then performed in T24 cells, during which tumor cells $\left(1 \times 10^{5}\right)$ were seeded into 24 -well plates and grown overnight. On the following day, $100 \mathrm{ng}$ luciferase reporter vectors and $100 \mathrm{nM}$ miR-612 or miR-NC were transfected into the T24 cells using Lipofectamine ${ }^{\circledR} 2000$ (Invitrogen; Thermo Fisher Scientific, Inc.) for $48 \mathrm{~h}$. Thereafter, the cell protein was extracted and the luciferase activity was measured using a luciferase reporter assay system (Promega Corporation), according to the manufacturer's protocol.

Western blot analysis. Total cellular protein was extracted from the transfected cells using a radioimmunoprecipitation assay buffer (Sigma-Aldrich) containing protease inhibitor, and the protein concentration of these samples was measured using a bicinchoninic acid assay kit (Thermo Fisher Scientific, Inc.) according to the manufacturer's protocol. Protein samples of $20 \mu \mathrm{g}$ each were separated with $10 \%$ sodium dodecyl sulfatepolyacrylamide gels by electrophoresis and then blotted onto nitrocellulose membranes (Sigma-Aldrich; Merck KGaA). For western blotting, the membranes were blocked with $5 \%$ non-fat milk for $1 \mathrm{~h}$ at $37^{\circ} \mathrm{C}$ and immunoblotted at $4^{\circ} \mathrm{C}$ overnight with primary antibodies against: ME1 (sc-365891, 1:1000, secondary antibodies: Mouse), E-cadherin (sc-52327, 1:1000 secondary antibodies: Mouse), N-cadherin (sc-53488, 1:1000, secondary antibodies: Mouse), vimentin (sc-373717, 1:1000, secondary antibodies: Mouse), matrix metalloproteinase-9 (MMP-9) (sc-12759, 1:1,000, secondary antibodies: Mouse), and anti- $\beta$-actin (sc-47778, 1:1000, secondary antibodies: Mouse) (all purchased from Santa Cruz Biotechnology, Dallas, TX, USA). The membranes were subsequently incubated with the fluoresence-conjugated secondary antibodies (926-68072 or 926-32210, LI-COR Biosciences, Shanghai, China) for $1 \mathrm{~h}$ at room temperature, and the protein bands were visualized and quantified by using the Odyssey two-color infrared laser imaging system (LI-COR Biosciences, Lincoln, NE, USA). 
A
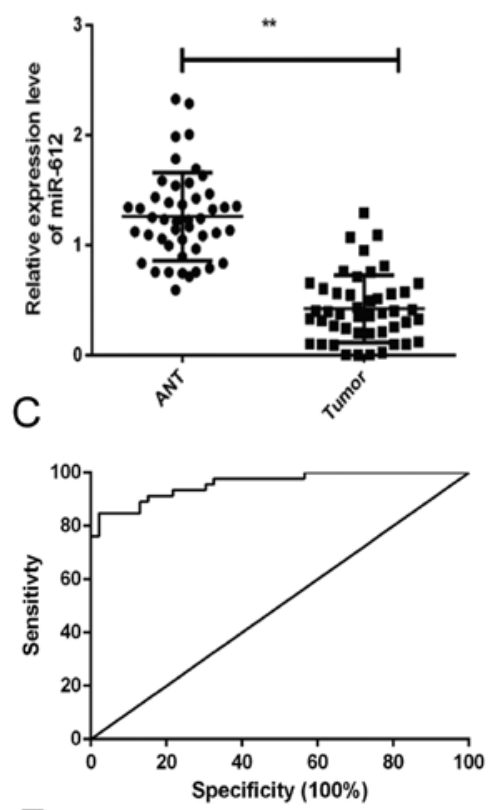

B

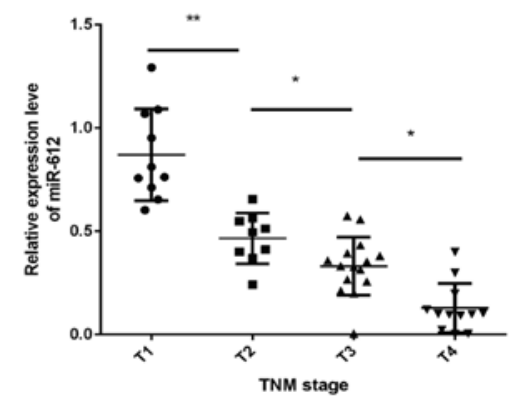

D

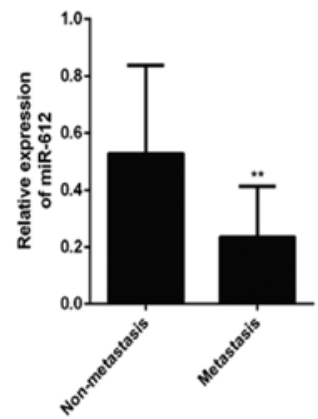

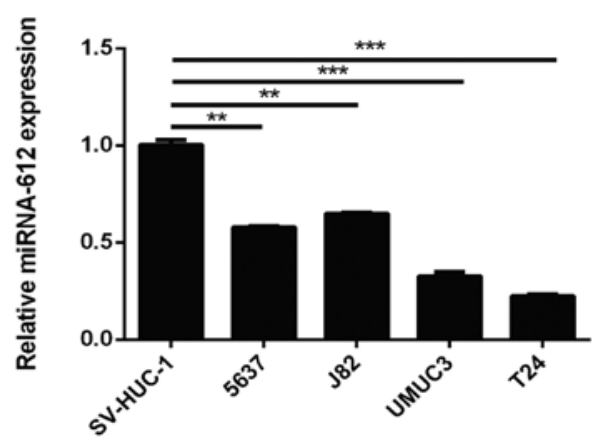

Figure 1. Expression and association of miR-612 with advanced TNM stages and distant tumor metastasis. (A) The aberrant miR-612 expression was assessed in bladder cancer and ANT specimens obtained from 46 patients using RT-qPCR. Association of the relative miR-612 expression in the bladder cancer tissue samples with (B) different TNM stages (miR-612 levels were normalized to the mean value of group T1; $\mathrm{n} \geq 9$ per group) and (C) distant tumor metastasis is displayed. (D) Receiver operating characteristic analysis, demonstrating that tissue miR-612 levels were able to distinguish bladder cancer patients from healthy controls. (E) miR-612 expression was detected in four bladder cancer cell lines (5637, J82, UMUC3 and T24) and the immortalized normal human bladder epithelial cell line SV-HUC-1 by RT-qPCR. ${ }^{*} \mathrm{P}<0.05,{ }^{* * *} \mathrm{P}<0.01$ and ${ }^{* * * *} \mathrm{P}<0.001$. miR, microRNA; ANT, adjacent normal tissue; RT-qPCR, reverse transcription-quantitative polymerase chain reaction.

Animal experiments. The animal experiments conducted in the present study were approved by the Animal Care and Use Committee of Tongji University. Briefly, 6 male nude BALB/c mice (age, 6 weeks old; weight, $18-22 \mathrm{~g}$ ) were purchased from Shanghai SIPPR-Bk Lab Animal Co., Ltd. (Shanghai, China) and housed in specific-pathogen-free conditions with a 12-h light/dark cycle (temperature, $18-22^{\circ} \mathrm{C}$; humidity, 50-60; irradiated feed and double-distilled water). Bladder cancer T24 cells stably expressing miR-612 or miR-NC were harvested, washed twice with PBS and resuspended in PBS. A total of $5 \times 10^{6}$ cells were then subcutaneously injected into the left flank regions of each nude mouse. Tumor cell xenograft formation was measured every 5 days using a Vernier caliper, and the tumor volume was calculated using the following formula: Volume $\left(\mathrm{mm}^{3}\right)=0.5 \mathrm{x}$ width $^{2} \mathrm{x}$ length. At 5 weeks after tumor cell inoculation, the mice were sacrificed by cervical dislocation. Tumor xenografts were resected, weighed and then stored at $-80^{\circ} \mathrm{C}$ until further use.

Statistical analysis. SPSS software (version 16.0; SPSS, Inc., Chicago, IL, USA) was used for all statistical analyses.
The experimental data were statistically analyzed using the Student's t-test or one-way analysis of variance, and are expressed as the mean \pm standard deviation from three independent experiments. The $\chi^{2}$ test or Fisher's exact test was performed to examine the correlation of miR-612 expression with the clinicopathological data of bladder cancer patients, whereas the association of miR-612 with ME1 expression was statistically analyzed using Spearman's correlation test. $\mathrm{P}<0.05$ was regarded to indicate a statistically significant difference.

\section{Results}

Downregulation of miR-612 expression in bladder cancer tissues and cell lines. In the present study, the levels of miR-612 expression in 46 pairs of bladder cancer and ANT specimens were initially assessed using RT-qPCR. It was observed that the miR-612 expression level was significantly lower in bladder cancer tissues compared with that in ANTs (P<0.01; Fig. 1A). Next, the association of miR-612 expression with the clinicopathological features of the patients was examined, and loss of miR-612 expression was 
A

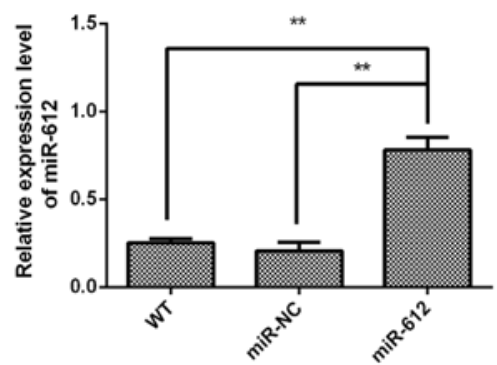

C

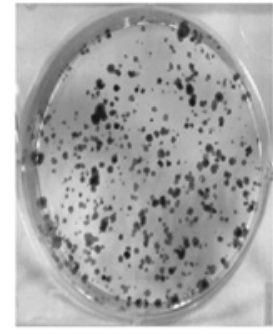

miR-NC

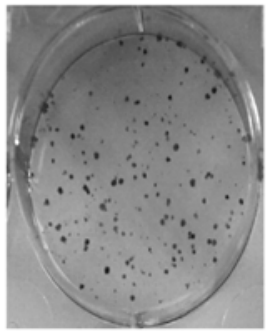

miR-612
D

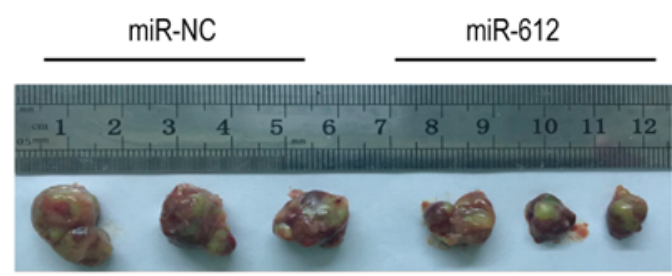

$\mathrm{B}$
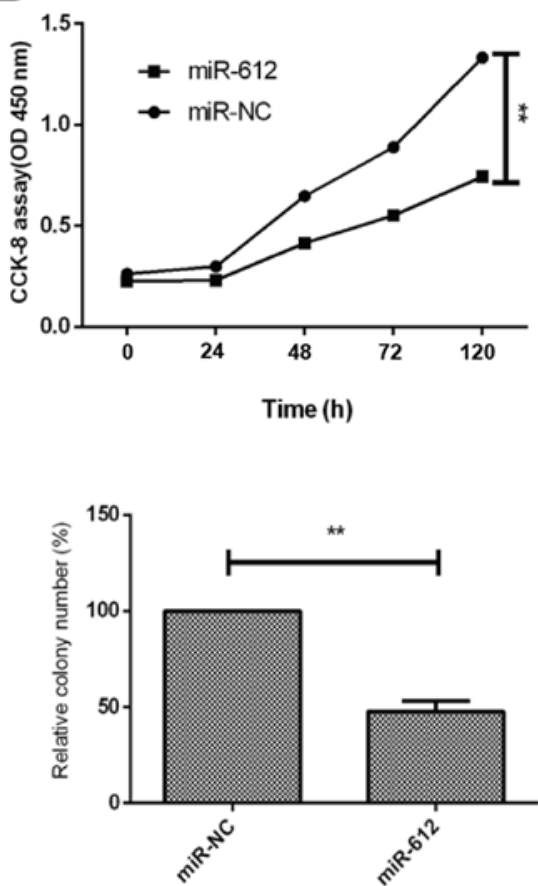

E

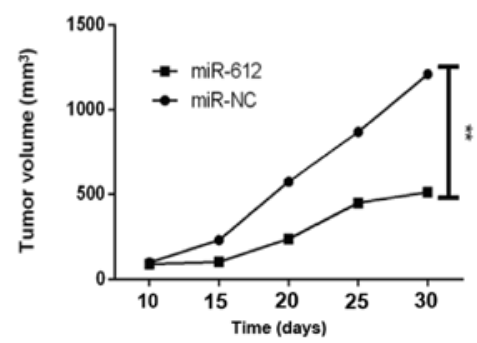

$\mathrm{F}$

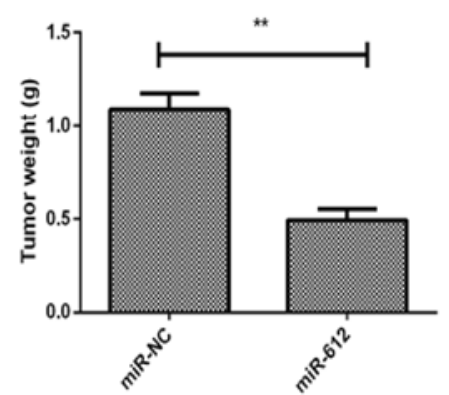

Figure 2. Effects of the miR-612 overexpression on the inhibition of bladder cancer cell proliferation in vitro and in a nude mouse xenograft assay. (A) miR-612 expression was detected in T24 cells following miR-612 mimic or miR-NC transfection and untransfected (WT) cells using reverse transcription-quantitative polymerase chain reaction. (B) Tumor cell growth and (C) colony formation were determined in T24 cells following transfection with miR-612 mimic or miR-NC by the CCK-8 assay. (D) Tumor xenograft growth, and (E) tumor xenograft weight of the miR-612 and miR-NC groups. (F) Nude mouse xenograft assay, showing images of tumor-carrying mice and tumor xenografts. The mice were divided into 2 groups ( 3 mice in each group). ${ }^{* *} \mathrm{P}<0.01$. miR, microRNA; $\mathrm{NC}$, negative control.

found to be significantly associated with advanced TNM stages (Fig. 1B) and tumor distant metastasis (Fig. 1D). Furthermore, the receiver operating characteristic curve analysis demonstrated that tissue miR-612 levels may be a potential biomarker for distinguishing bladder cancer patients from the controls (area under the curve, 0.958; Fig. 1C). Consistently, miR-612 expression was also significantly lower in T24, UMUC3, 5637 and J82 bladder cancer cells as compared with that in the normal bladder epithelial cell line SV-HUC-1. T24 cells exhibited the lowest miR-612 levels among these four bladder cancer cell lines (Fig. 1E).
Inhibition of bladder cancer growth following miR-612 overexpression in vitro and in vivo. To determine the biological effects of miR-612 on bladder cancer cell growth, miR-612 mimic or negative control was transfected into T24 cells, since this cell line had the lowest level of miR-612 expression (Fig. 1E). The data revealed that the miR-612 mimic was successfully transfected into the cells and increased miR-612 expression; miR-NC transfection had no significant effect on the T24 cells (Fig. 2A). The CCK-8 and colony formation assays demonstrated that miR-612 overexpression in 
A

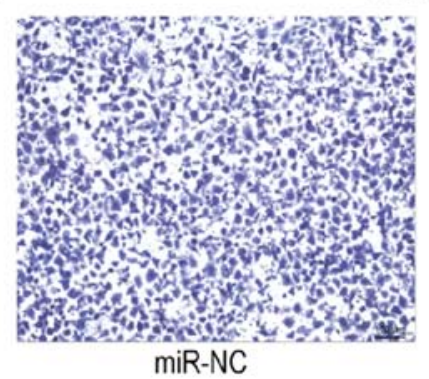

Migration

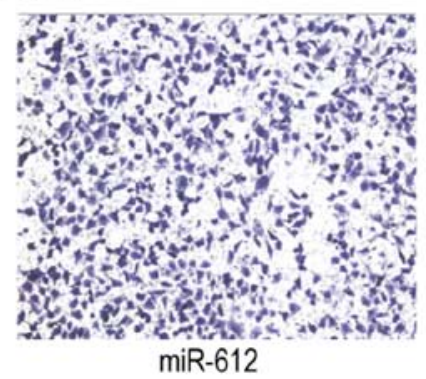

B

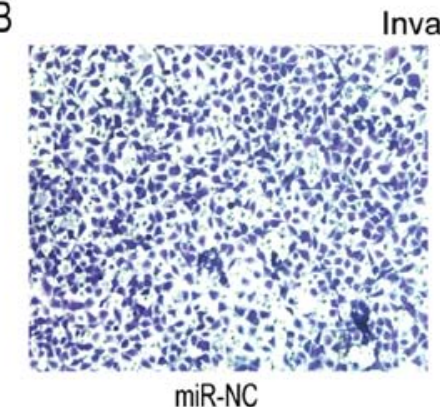

Invasion

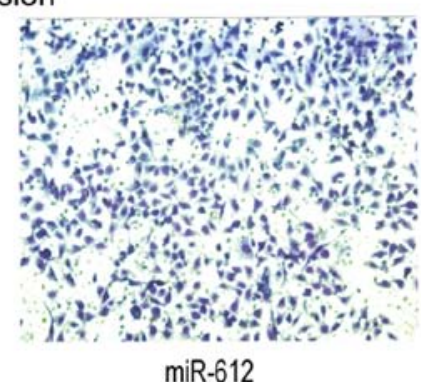

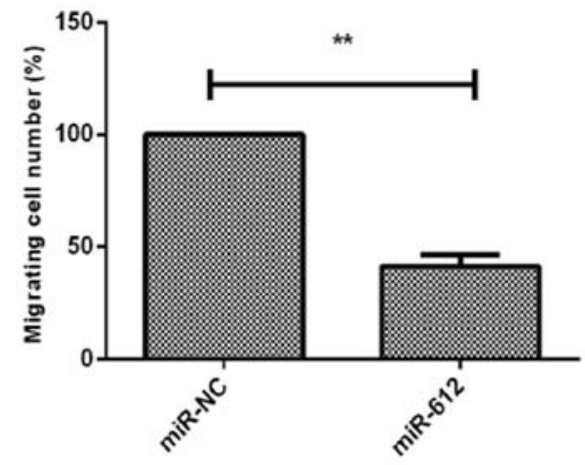

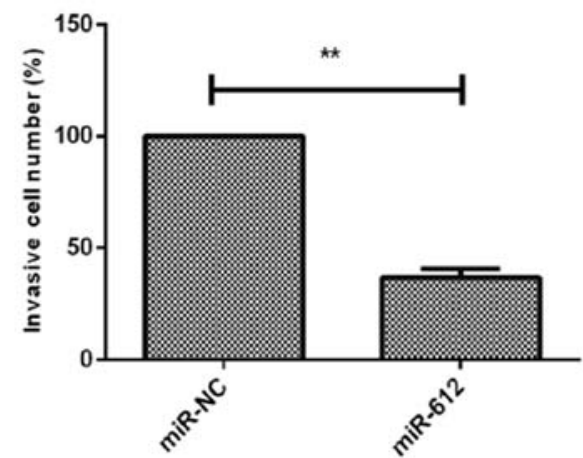

C
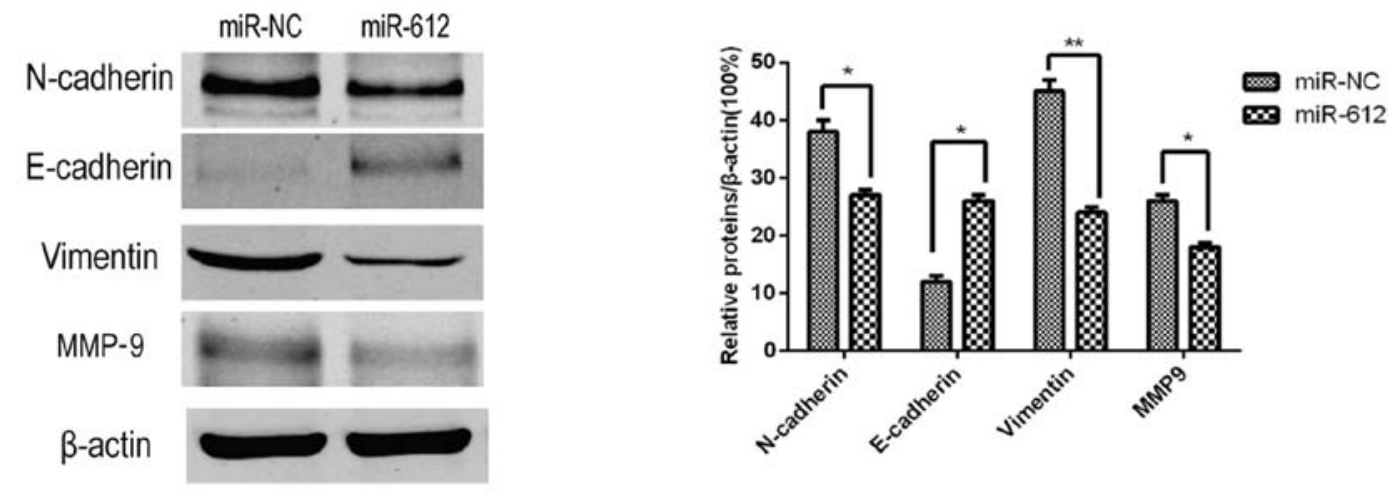

Figure 3. Effects of the miR-612 overexpression on the inhibition of bladder cancer cell migration, invasion and EMT. (A) Migration and (B) invasion abilities of T24 cells following transfection with miR-612 or miR-NC was detected by Transwell tumor cell migration and invasion assays, respectively. (C) Expression levels of EMT-associated markers in T24 cells following transfection with miR-612 mimic or miR-NC were evaluated by western blot analysis. ${ }^{*} \mathrm{P}<0.05$ and ${ }^{* *} \mathrm{P}<0.01$. miR, microRNA; NC, negative control; EMT, epithelial-mesenchymal transition; MMP-9, matrix metalloproteinase-9.

T24 cells significantly reduced tumor cell growth (Fig. 2B) and colony formation (Fig. 2C). Furthermore, the nude mouse experiments indicated that T24 cells with miR-612 overexpression resulted in slower growth of the tumor xenografts as compared with that for miR-NC-transfected T24 cells (Fig. 2E). Consistently, the weight (Fig. 2F) and tumor xenograft size (Fig. 2D) of the miR-612-transfected group were significantly reduced in comparison with those of the miR-NC-transfected group.

miR-612 inhibition of tumor cell migration, invasion and EMT in vitro. Next, the effects of miR-612 on the metastatic capacity of bladder cancer cells were assessed in vitro, and it was observed that restoration of miR-612 expression in T24 cells using the miR-612 mimic significantly reduced the tumor cell migration and invasion abilities in vitro (Fig. 3A and B). Furthermore, the expression of several accepted EMT-associated markers was also affected by the miR-612 mimic. Compared with the
miR-NC group, the expression levels of N-cadherin, vimentin and MMP-9 were significantly decreased by miR-612 mimic transfection, whereas the expression level of E-cadherin was significantly upregulated in the miR-612 group (Fig. 3C). During EMT, N-cadherin, vimentin and MMP-9 levels are upregulated, and E-cadherin levels are downregulated. Thus, our findings indicated that miR-612 overexpression inhibited EMT.

ME1 is a the direct target of miR-612. Since miRNAs alter the expression of their target genes to exert their biological functions in cells, bioinformatic analysis was performed using several software (DIANAmT, miRanda, miRDB, miRWalk, PICTAR5, RNA22 and Targetscan) to predict the possible targets of miR-612. Subsequently, relevant publications on these genes were retrieved from Pubmed, and genes that have been widely studied in bladder cancer and were not highly expressed in other tumors were excluded. Finally, it was 
A

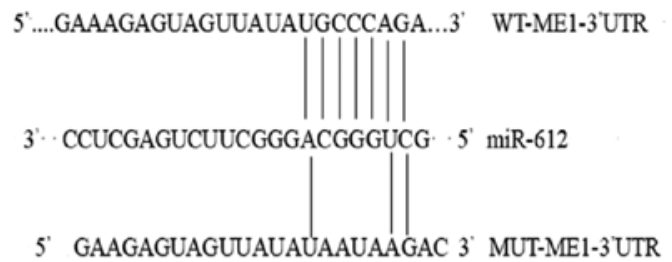

C

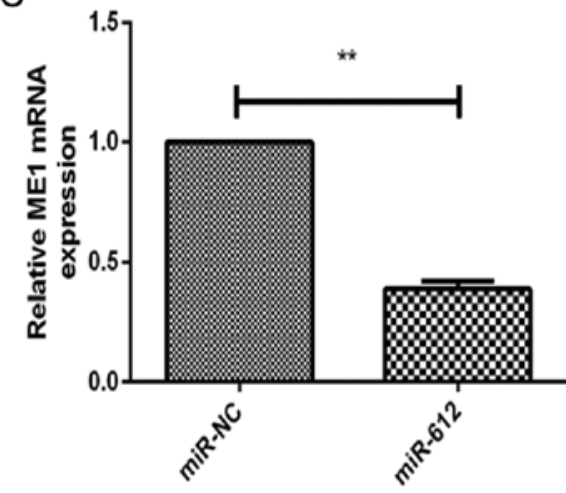

E

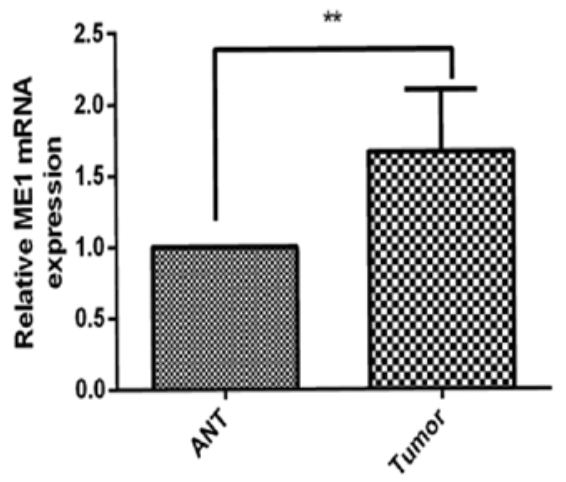

B

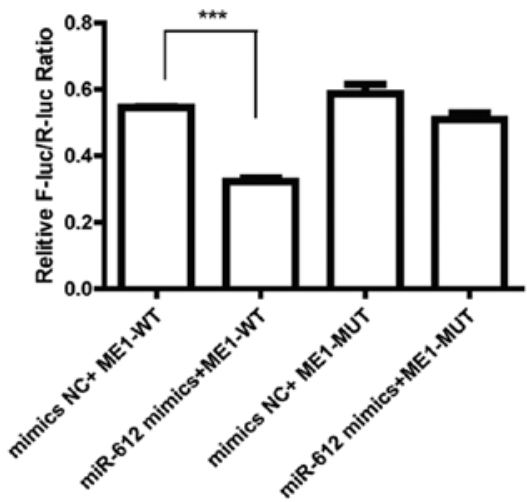

D

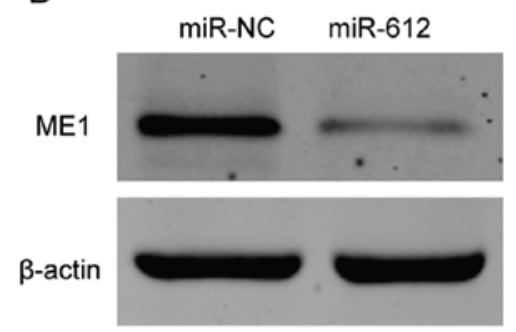

$\mathrm{F}$

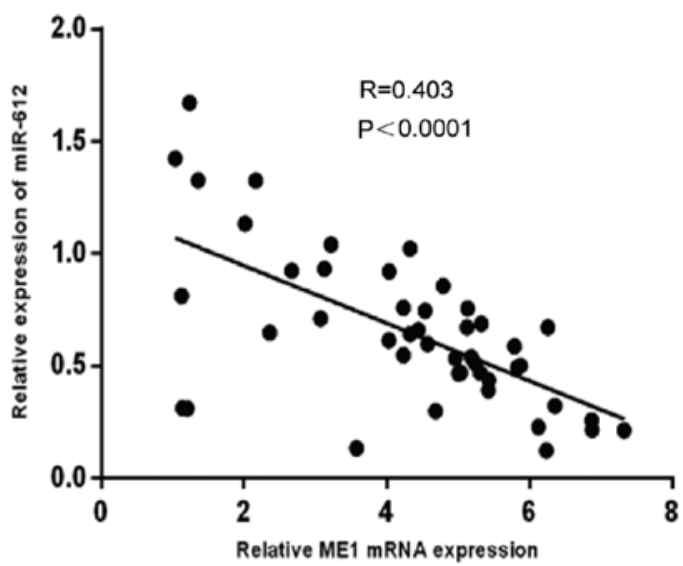

Figure 4. ME1 is a direct target of miR-612. (A) Bioinformatic analysis indicated that ME1 is the target of miR-612. The presumable miR-612-binding sites in the 3'-UTR of ME1 cDNA and the mutant binding sites are shown. (B) The relative luciferase activity was determined in T24 cells following co-transfection with a luciferase reporter plasmid (WT or MUT 3'-UTR ME1 cDNA), and miR-612 mimic or miR-NC. (C) mRNA and (D) protein levels of ME1 in T24 cells following transfection with miR-612 mimic or miR-NC were analyzed using RT-qPCR and western blot analysis, respectively. GAPDH and $\beta$-actin served as the internal controls, respectively. (E) The levels of ME1 mRNA were measured using RT-qPCR in 46 paired bladder cancer and ANT specimens. GAPDH was used as an internal control. (F) The correlation between ME1 and miR-612 expression in bladder cancer tissue samples (n=46) was analyzed using Spearman's correlation test. ${ }^{* *} \mathrm{P}<0.01$ and ${ }^{* * *} \mathrm{P}<0.001$. miR, microRNA; NC, negative control; ME1, malic enzyme 1; UTR, untranslated region; WT, wild-type; MUT, mutated; ANT, adjacent normal tissue; RT-qPCR, reverse transcription-quantitative polymerase chain reaction.

detected that miR-612 was able to bind to the 3'-UTR of ME1 cDNA (Fig. 4A). This binding ability of miR-612 to the ME1 3'-UTR was then confirmed using a luciferase reporter assay. The results revealed that miR-612 was able to precisely bind to the wild-type 3'-UTR of ME1, which resulted in significantly inhibited luciferase activity (Fig. 4B). Furthermore, it was demonstrated that miR-612 overexpression in T24 cells significantly downregulated the mRNA (Fig. 4C) and protein (Fig. 4D) levels of ME1. The ex vivo data further supported this observation, since the level of ME1 mRNA was markedly higher in bladder cancer tissues as compared with that in ANTs (Fig. 4E). In addition, the level of ME1 mRNA was inversely associated with the level of miR-612 in bladder cancer tissues (Fig. 4F; r=-0.403; $\mathrm{P}<0.0001$ ). These results indicated that ME1 is indeed the direct target of miR-612 in bladder cancer.

Parallel effects of ME1 knockdown and overexpression with miR-612 manipulation on bladder cancer cells in vitro. To further confirm the effect and biological functions of ME1 in bladder cancer cells, ME1 expression was knocked down in T24 cells using si-ME1 and compared against the si-NC group. The data demonstrated that si-ME1 significantly reduced the ME1 mRNA and protein expression levels in T24 cells, as compared with the miR-NC-transfected T24 cells (Fig. 5A and B). In addition, functional assays revealed that ME1 knockdown significantly inhibited the tumor cell growth (Fig. 5C), colony formation (Fig. 5D), migration (Fig. 5E) and 
A

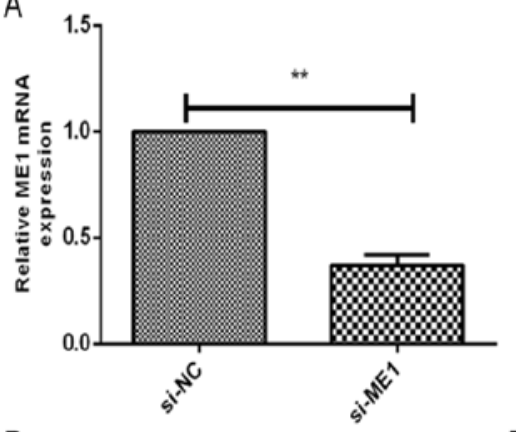

D

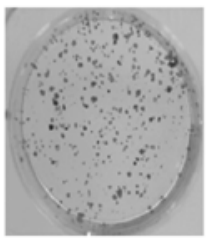

si-NC

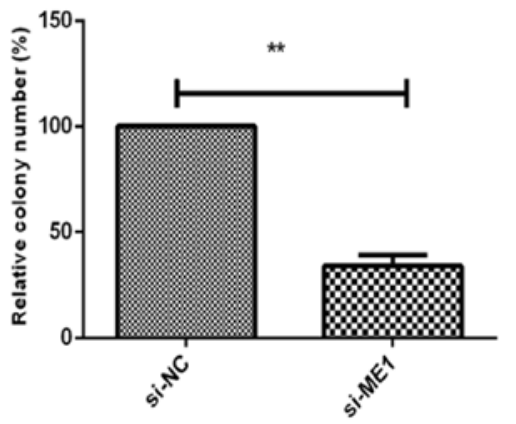

G
B

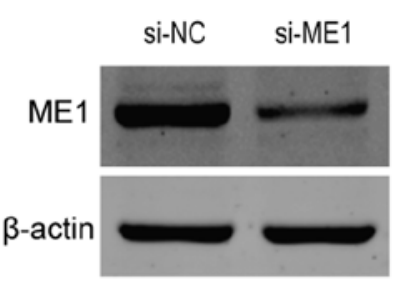

$\mathrm{E}$
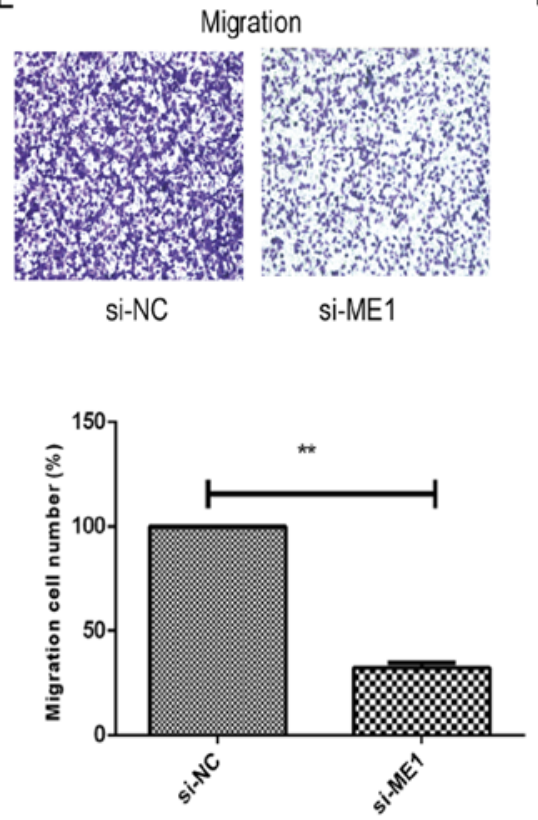

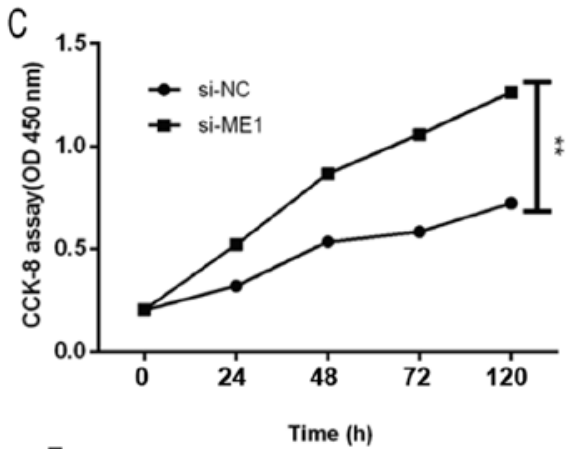

$\mathrm{F}$

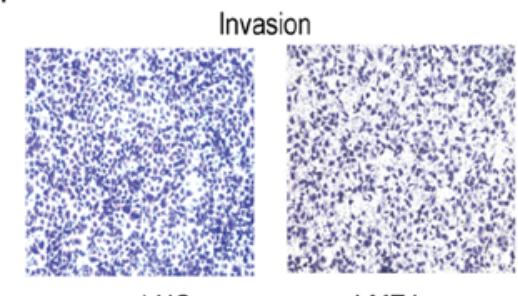

si-NC

Si-ME1

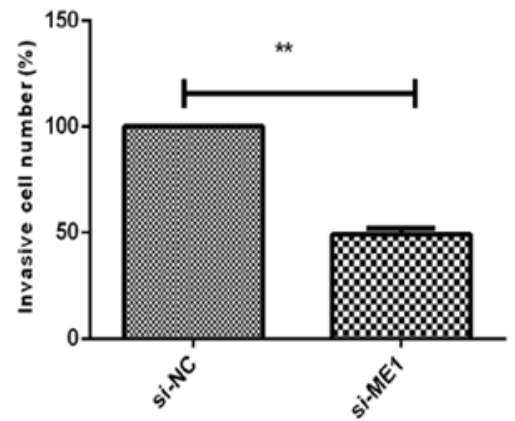

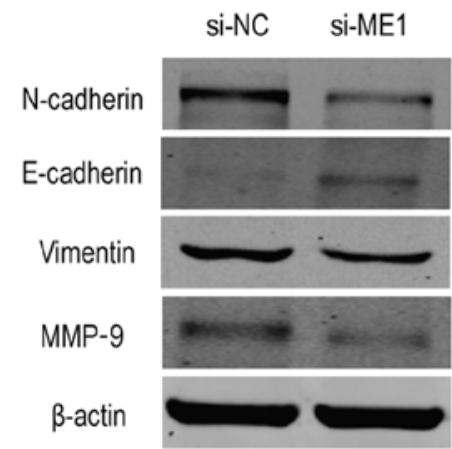

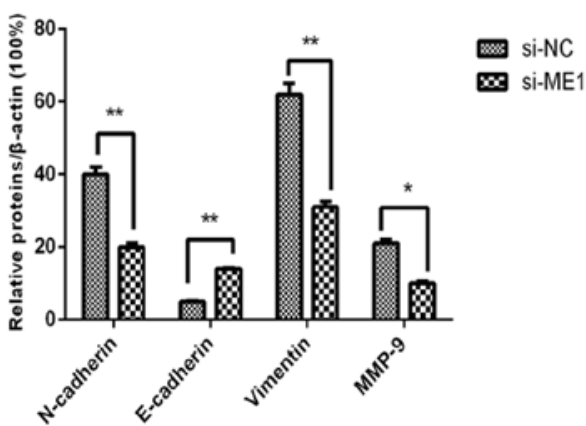

Figure 5. ME1 knockdown mimicked the inhibitory effect of miR-612 overexpression in bladder cancer cells. (A) mRNA and (B) protein levels of ME1 in T24 cells following transfection with si-ME1 or si-NC were measured using reverse transcription-quantitative polymerase chain reaction and western blot analysis, respectively. GAPDH and $\beta$-actin were used as internal controls, respectively. (C) Cell proliferation, (D) colony formation, (E) Transwell tumor cell migration assay and (F) Transwell tumor cell invasion assays in bladder cancer T24 cells transfected with si-ME1 or si-NC. (G) Protein levels of EMT-associated markers in $\mathrm{T} 24$ cells transfected with si-ME1 or si-NC were evaluated by western blot analysis. " $\mathrm{P}<0.05$ and ${ }^{* *} \mathrm{P}<0.01$. miR, microRNA; NC, negative control; ME1, malic enzyme 1 ; si, siRNA.

invasion capacity (Fig. 5F), and affected the tumor cell EMT marker levels (Fig. 5G) in T24 cells, mimicking the effects of miR-612 overexpression on T24 cells (Figs. 2 and 3).

Furthermore, to better verify that ME1 was a direct target of miR-612, the T24 cells were simultaneously co-transfected with the miR-612 mimic or miR-NC and ME1 overexpression plasmid. We found that ME1 overexpression using ME1 cDNA transfection in miR-612-expressing T24 cells was able to partially reverse miR-612 overexpression-induced reduction of ME1 expression in T24 cells (Fig. 6A and B). The ME1 overexpression also functionally rescued the inhibitory effect of miR-612 overexpression on tumor cell growth, colony formation, migration and invasion (Fig. 6C-F). Taken together, the results indicated that miR-612 inhibited tumor cell growth, colony formation, migration, invasion and EMT by targeting ME1 in vitro (Fig. 7). 

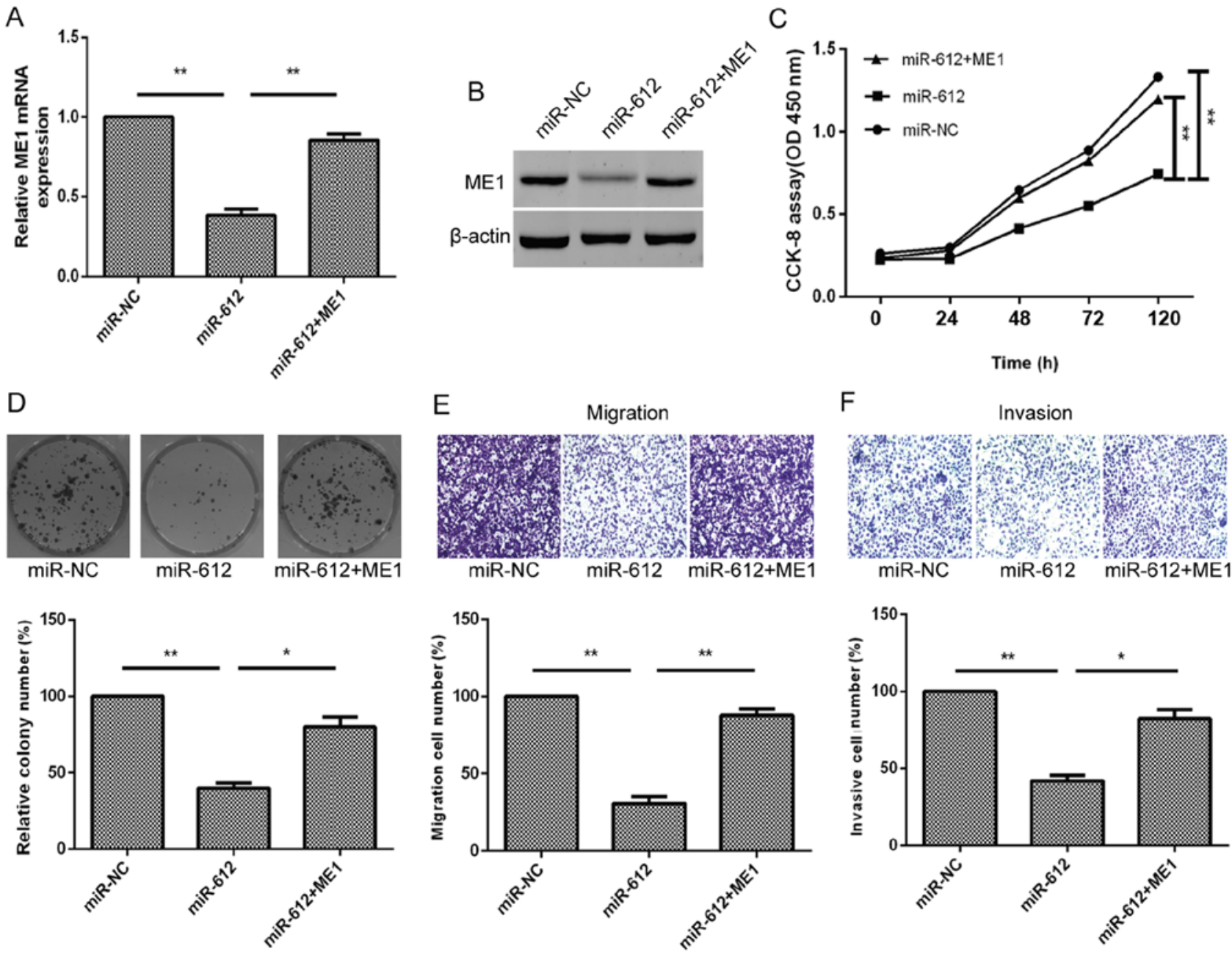

Figure 6. ME1 overexpression partially rescues the inhibitory effects of miR-612 expression on bladder cancer cells. (A) mRNA and (B) protein levels of ME1 in T24 cells co-transfected with miR-612 mimic or miR-NC, and with or without ME1 cDNA were measured using reverse transcription-quantitative polymerase chain reaction and western blot analysis, respectively. (C) Cell proliferation, (D) colony formation, (E) Transwell tumor cell migration and (F) Transwell tumor cell invasion assays in T24 cells co-transfected with miR-612 mimic or miR-NC, and with or without ME1 cDNA. ${ }^{*} \mathrm{P}<0.05$ and ${ }^{* *} \mathrm{P}<0.01$. miR, microRNA; NC, negative control; ME1, malic enzyme 1; si, siRNA.

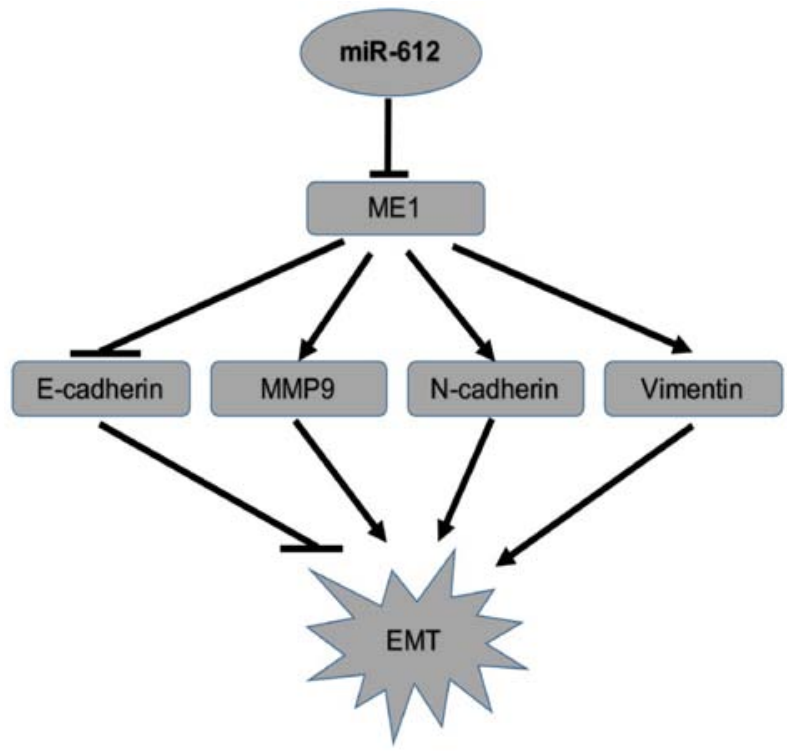

Figure 7. Illustration of the proposed model for the mechanistic interaction of ME1 with miR-612 in the regulation of the tumor cell EMT. miR, microRNA; NC, negative control; EMT, epithelial-mesenchymal transition; ME1, malic enzyme 1 .

\section{Discussion}

Accumulating evidence has demonstrated that aberrant miRNA expression contributes to the development and progression of human cancer, including bladder cancer (30-34). The present study further confirmed this notion for miR-612 in bladder cancer tissues and cell lines. It was observed that miR-612 expression was downregulated in bladder cancer tissues and cell lines as compared with that in normal tissue and cells. The data also revealed that loss of miR-162 expression was associated with advanced bladder cancer TNM stages and metastasis. Additionally, it was demonstrated that miR-612 suppressed bladder cancer cell proliferation in vitro and in vivo by targeting ME1 expression. These results suggest that miR-612 may be a tumor suppressor gene or possess tumor-suppressing functions in bladder cancer. Further studies will investigate whether miR-612 serves as a potential tumor biomarker or therapeutic target for bladder cancer.

Previous studies have reported that altered miR-612 expression is involved in the development and progression of many types of human cancer, including hepatocellular carcinoma and colorectal cancer. For instance, miR-612 expression was 
able to inhibit liver cancer stemness and target AKT2 expression via the $\mathrm{Wnt} / \beta$-catenin signaling pathway (19). In addition, miR-612 expression suppressed colorectal cancer growth and metastasis by targeting AKT2 expression (20), while miR-612 was shown to serve an inhibitory effect on hepatocellular carcinoma cell stemness through modulation of the Sp1/Nanog signaling pathway (18). These previous findings suggest that miR-612 functions as a putative tumor suppressor in the aforementioned types of cancer. Furthermore, the present study demonstrated low expression of miR-612 in bladder cancer tissues and cell lines; however, the target gene ME1 was highly expressed in bladder cancer tissues. This indicated that, while miR-612 serves as a tumor suppressor in bladder cancer, the target gene ME1 was cancer-dependent. Further studies should investigate the association between miR-612 and the target genes possessing miR-612 tumor-suppressing functions in human cancer.

In the present study, the effects of miR-612 in bladder cancer were found to occur via the targeting of ME1 expression, according to the following observations: i) High expression of ME1 mRNA was inversely associated with miR-612 expression in bladder cancer tissues; ii) miR-612 was able to bind to the 3'-UTR of ME1 cDNA, but not to the mutated ME1 molecule; iii) miR-612 mimic transfection was able to downregulate ME1 expression; iv) ME1 knockdown significantly inhibited tumor cell growth, colony formation, and migration and invasion capacities, which mimicked the effects of miR-612 overexpression on T24 cells; and v) ME1 overexpression in miR-612-expressing T24 cells partially reversed miR-612 overexpression-reduced ME1 expression and functionally rescued the inhibitory effect of miR-612 overexpression on tumor cell growth, colony formation, migration and invasion.

ME1 is a cytosolic $\mathrm{NADP}^{+}$-dependent enzyme this is critically responsible for NADPH production, fatty acid biosynthesis, and lipogenesis in cells and tissues (35). ME1 expression is upregulated in various types of human cancer, such as in colorectal cancer, and is associated with a poor overall survival of patients $(36,37)$. It was also reported that ME1 expression induces EMT of hepatocellular carcinoma cells and is associated with a poor prognosis of these patients (25). In addition, knockdown of ME1 expression has been demonstrated to inhibit the nasopharyngeal carcinoma cell migration and invasion capacity. Another study reported that mutant KRAS was associated with ME1 overexpression and predicted the response of non-small cell lung cancer cells to radiation therapy (38). Furthermore, the p53 protein reciprocally regulated ME1 expression to modulate cell metabolism and senescence (35). To the best of our knowledge, the present study demonstrated for the first time that the levels of ME1 mRNA were upregulated in bladder cancer tissues, but inversely associated with miR-612 expression. Meanwhile, the in vivo data revealed that miR-612 suppressed the growth of bladder cancer cell-induced xenografts in mice by repression of ME1 expression.

In conclusion, the current results provided evidence indicating that miR-612 expression is downregulated in bladder cancer tissues and cell lines, and that decreased miR-612 expression is associated with advanced bladder cancer TNM stages and distant metastasis. The in vitro and in vivo data further suggested that miR-612 expression was able to inhibit bladder cancer cell growth, colony formation, migration, invasion and EMT through directly targeting the expression of ME1. These findings indicate that miR-612 may serve as a novel therapeutic target for bladder cancer.

\section{Acknowledgements}

The authors would like to thank the personnel of the Central Laboratory of the Shanghai Tenth People's Hospital for their assistance and support.

\section{Funding}

This study was supported in part by a grant from the National Natural Science Foundation of China (no. 81272836).

\section{Availability of data and materials}

The analyzed datasets generated during the study are available from the corresponding author on reasonable request.

\section{Authors' contributions}

ML and XY made substantial contributions to the conception and design of the study; ML and YC drafted the manuscirpt; ML made substantial contributions to the acquisition, analysis, and interpretation of the data for the study; YC made substantial contributions to the interpretation of the data for the study; $\mathrm{BH}$ contributed to the design of the study; SM, KC, LW and $\mathrm{XY}$ contributed to the acquisition and analysis of the data for the study; $\mathrm{BH}, \mathrm{SM}, \mathrm{KC}, \mathrm{LW}$ and $\mathrm{XY}$ revised the manuscript critically for important intellectual content. All authors gave the final approval of the manuscript to be published and agree to be accountable for all aspects of the study in ensuring that questions related to the accuracy or integrity of any part of the study are appropriately investigated and resolved.

\section{Ethics approval and consent to participate}

The patient study was approved by the Ethics Committee of Shanghai Tenth People's Hospital of Tongji University. Informed consent was obtained from all patients or their relatives. The animal experiments were approved by the Animal Care and Use Committee of Tongji University.

\section{Consent for publication}

Not applicable.

\section{Competing interests}

The authors declare that they have no competing interests

\section{References}

1. McGuire S: World Cancer Report 2014. Geneva, Switzerland: World Health Organization, International Agency for Research on Cancer, WHO Press, 2015. Adv Nutr 7: 418-419, 2016.

2. Torre LA, Bray F, Siegel RL, Ferlay J, Lortet-Tieulent J and Jemal A: Global cancer statistics, 2012. CA Cancer J Clin 65: 87-108, 2015

3. Siegel RL, Miller KD and Jemal A: Cancer Statistics, 2017. CA Cancer J Clin 67: 7-30, 2017. 
4. Zeng LP, Hu ZM, Li K and Xia K: miR-222 attenuates cisplatininduced cell death by targeting the PPP2R 2A/Akt/mTOR Axis in bladder cancer cells. J Cell Mol Med 20: 559-567, 2016.

5. Yang X, Cheng Y, Li P, Tao J, Deng X, Zhang X, Gu M, Lu Q and Yin C: A lentiviral sponge for miRNA-21 diminishes aerobic glycolysis in bladder cancer T24 cells via the PTEN/PI3K/AKT/ mTOR axis. Tumour Biol 36: 383-391, 2015.

6. Hanke M, Hoefig K, Merz H, Feller AC, Kausch I, Jocham D, Warnecke JM and Sczakiel G: A robust methodology to study urine microRNA as tumor marker: microRNA-126 and microRNA-182 are related to urinary bladder cancer. Urol Oncol 28: 655-661, 2010

7. Bushati N and Cohen SM: microRNA functions. Annu Rev Cell Dev Biol 23: 175-205, 2007.

8. Hwang HW and Mendell JT: MicroRNAs in cell proliferation, cell death, and tumorigenesis. Br J Cancer 96 (Suppl): R40-R44, 2007.

9. Calin GA and Croce CM: MicroRNA signatures in human cancers. Nat Rev Cancer 6: 857-866, 2006.

10. Vinall RL, Ripoll AZ, Wang S, Pan CX and deVere White RW: miR-34a chemosensitizes bladder cancer cells to cisplatin treatment regardless of $\mathrm{p} 53-\mathrm{Rb}$ pathway status. Int J Cancer 130 2526-2538, 2012.

11. Shen J, Zhang J, Xiao M, Yang J and Zhang N: miR-203 suppresses bladder cancer cell growth and targets the Twist1. Oncol Res: Sep 6, 2017 (Epub ahead of print). doi: 10.3727/0965 04017X15041934685237.print) 2017.

12. Pop-Bica C, Gulei D, Cojocneanu-Petric R, Braicu C, Petrut B and Berindan-Neagoe I: Understanding the role of non-coding RNAs in bladder cancer: From dark matter to valuable therapeutic targets. Int J Mol Sci 18: 18, 2017.

13. Wei Z, Hu X, Liu J, Zhu W, Zhan X and Sun S: MicroRNA-497 upregulation inhibits cell invasion and metastasis in T24 and BIU-87 bladder cancer cells. Mol Med Rep 16: 2055-2060, 2017.

14. Wu D, Niu X, Tao J, Li P, Lu Q, Xu A, Chen W and Wang Z: MicroRNA-379-5p plays a tumor-suppressive role in human bladder cancer growth and metastasis by directly targeting MDM2. Oncol Rep 37: 3502-3508, 2017.

15. Zhang Y, Zhang Z, Li Z, Gong D, Zhan B, Man X and Kong C: MicroRNA-497 inhibits the proliferation, migration and invasion of human bladder transitional cell carcinoma cells by targeting E2F3. Oncol Rep 36: 1293-1300, 2016.

16. Yu H, Duan P, Zhu H and Rao D: miR-613 inhibits bladder cancer proliferation and migration through targeting SphK1. Am J Transl Res 9: 1213-1221, 2017.

17. Wu CL, Ho JY, Chou SC and Yu DS: miR-429 reverses epithelialmesenchymal transition by restoring E-cadherin expression in bladder cancer. Oncotarget 7: 26593-26603, 2016.

18. Liu Y, Liu DL, Dong LL, Wen D, Shi DM, Zhou J, Fan J and Wu WZ: miR-612 suppresses stem cell-like property of hepatocellular carcinoma cells by modulating Sp1/Nanog signaling. Cell Death Dis 7: e2377, 2016.

19. Tang J, Tao ZH, Wen D, Wan JL, Liu DL, Zhang S, Cui JF, Sun HC, Wang L, Zhou J, et al: miR-612 suppresses the stemness of liver cancer via Wnt/ $\beta$-catenin signaling. Biochem Biophys Res Commun 447: 210-215, 2014.

20. Sheng L, He P, Yang X, Zhou M and Feng Q: miR-612 negatively regulates colorectal cancer growth and metastasis by targeting AKT2. Cell Death Dis 6: e1808, 2015.

21. Umetsu SE, Shafizadeh N and Kakar S: Grading and staging mucinous neoplasms of the appendix: A case series and review of the literature. Hum Pathol 69: 81-89, 2017.

22. Dweep H, Sticht C, Pandey P and Gretz N: miRWalk--database: Prediction of possible miRNA binding sites by 'walking' the genes of three genomes. J Biomed Inform 44: 839-847, 2011.
23. Dweep H and Gretz N: miRWalk2.0: A comprehensive atlas of microRNA-target interactions. Nat Methods 12: 697, 2015.

24. Hu H, Chen Y, Cheng S, Li G and Zhang Z: Dysregulated expression of homebox gene HOXA13 is correlated with the poor prognosis in bladder cancer. Wien Klin Wochenschr 129: 391-397, 2017.

25. Schenková K, Lutz J, Kopp M, Ramos S and Rivero F: MUF1/ leucine-rich repeat containing 41 (LRRC41), a substrate of RhoBTB-dependent cullin 3 ubiquitin ligase complexes, is a predominantly nuclear dimeric protein. J Mol Biol 422: 659-673, 2012.

26. Zheng H, Li Q, Chen R, Zhang J, Ran Y, He X, Li S and Shu HB: The dual-specificity phosphatase DUSP14 negatively regulates tumor necrosis factor- and interleukin-1-induced nuclear factor- $x \mathrm{~B}$ activation by dephosphorylating the protein kinase TAK1. J Biol Chem 288: 819-825, 2013.

27. Mukhtar YM, Huang Y, Liu J, Chen D and Zheng W: Acetanilide and bromoacetyl-lysine derivatives as activators for human histone deacetylase 8. Bioorg Med Chem Lett 27: 2319-2323, 2017.

28. Sengupta S, Weeraratne SD, Sun H, Phallen J, Rallapalli SK, Teider N, Kosaras B, Amani V, Pierre-Francois J, Tang Y, et al: a5-GABAA receptors negatively regulate MYC-amplified medulloblastoma growth. Acta Neuropathol 127: 593-603, 2014.

29. Livak KJ and Schmittgen TD: Analysis of relative gene expression data using real-time quantitative PCR and the 2(-Delta Delta C(T)) Method. Methods 25: 402-408, 2001

30. Braicu C, Cojocneanu-Petric R, Chira S, Truta A, Floares A, Petrut B, Achimas-Cadariu P and Berindan-Neagoe I: Clinical and pathological implications of miRNA in bladder cancer. Int $\mathrm{J}$ Nanomedicine 10: 791-800, 2015.

31. Yoshino H, Seki N, Itesako T, Chiyomaru T, Nakagawa M and Enokida H: Aberrant expression of microRNAs in bladder cancer. Nat Rev Urol 10: 396-404, 2013.

32. Hu Y, Cheng C, Hong Z and Shi Z: Independent prognostic miRNAs for bladder urothelial carcinoma. Oncol Lett 14: 3001-3005, 2017.

33. Liu X, Liu X, Wu Y, Wu Q, Wang Q, Yang Z and Li L: MicroRNAs in biofluids are novel tools for bladder cancer screening. Oncotarget 8: 32370-32379, 2017.

34. Wang X, Liang Z, Xu X, Li J, Zhu Y, Meng S, Li S, Wang S, Xie B, Ji A, et al: miR-148a-3p represses proliferation and EMT by establishing regulatory circuits between ERBB3/AKT2/c-myc and DNMT1 in bladder cancer. Cell Death Dis 7: e2503, 2016.

35. Jiang P, Du W, Mancuso A, Wellen KE and Yang X: Reciprocal regulation of p53 and malic enzymes modulates metabolism and senescence. Nature 493: 689-693, 2013.

36. Wen D, Liu D, Tang J, Dong L, Liu Y, Tao Z, Wan J, Gao D, Wang L, Sun H, et al: Malic enzyme 1 induces epithelial-mesenchymal transition and indicates poor prognosis in hepatocellular carcinoma. Tumour Biol 36: 6211-6221, 2015.

37. Shen H, Xing C, Cui K, Li Y, Zhang J, Du R, Zhang X and Li Y: MicroRNA-30a attenuates mutant KRAS-driven colorectal tumorigenesis via direct suppression of ME1. Cell Death Differ 24: 1253-1262, 2017

38. Chakrabarti G: Mutant KRAS associated malic enzyme 1 expression is a predictive marker for radiation therapy response in non-small cell lung cancer. Radiat Oncol 10: 145, 2015.

This work is licensed under a Creative Commons Attribution-NonCommercial-NoDerivatives 4.0 International (CC BY-NC-ND 4.0) License. 